\title{
Mental Simulation as a Remedy for Biased Reasoning
}

\author{
Daša Strachanová, Lenka Valuš \\ Institute of Experimental Psychology, Center of Social and Psychological Sciences \\ Slovak Academy of Sciences
}

\begin{abstract}
Prompting mental simulation with a counterfactual scenario has been found to enhance rationality in individuals and groups. Building upon previous findings and the dual-process accounts of reasoning, we hypothesized that debiasing power of mental simulation lies in inhibiting System 1 and facilitating System 2 responses. Therefore, we examined whether counterfactual priming mitigates biased reasoning via changes in cognitive reflection. Each participant of our between-subject experiment $(N=462)$ solved two out of three tasks on biased reasoning: one before and one after being exposed to the counterfactual scenario. The tasks were designed to elicit selectively seeking hypothesis-confirming evidence, ignoring alternative explanations, and unwillingness to reconsider the default option. In addition, the participants completed two sets of cognitive reflection problems at the beginning and at the end of the experiment. Mental simulation reduced people's tendencies to ignore alternative explanations and hypothesisdisconfirming evidence, and the latter effect was mediated by intuition inhibition.
\end{abstract}

Key words: debiasing; counterfactual priming; mental simulation; cognitive reflection

\section{Introduction}

Decades of research have focused on documenting all the deviations from optimal judgments and choices people fall prey to. Lists of these cognitive biases seem endless (see Benson, 2016, for a nice visualization), and we moved from the concept of so-called "homo economicus" to a much more realistic image of human reasoning. As a result, finding ways of

Acknowledgment

This study was funded by the Slovak Research and Development Agency and is part of research project APVV-16-0153 "Cognitive failures - individual predictors and intervention possibilities".

Correspondence concerning this paper should be addressed to Lenka Valuš, Institute of Experimental Psychology, Center of Social and Psychological Sciences, Slovak Academy of Sciences, Dúbravská cesta 9, Bratislava, 84104, Slovak Republic. E-mail: valus.lenka@gmail.com

Received November 13, 2018 improving decision-making, especially when high stakes are at play, is being widely discussed. This, however, proved to be a much bigger challenge than we might have expected. Early debiasing attempts based on warnings about the possibility of bias occurrence, explaining nature of the bias, and providing a dose of feedback failed to succeed (Fischhoff, 1982). How far have we got, a quarter century later?

In the current debiasing efforts, two approaches have been applied: we either try to change the environment or the decision-maker. Typical examples of the first category are nudge techniques (Thaler\& Sunstein, 2008), helpful external representations (e.g., Sirota, Kostovičová, \& Juanchich, 2014) or inducing the sense of accountability(Lerner \& Tetlock, 1999). Soll, Milkman, and Payne (2016) list various other possibilities for modifying the environment in favor of better decisions. The other way, based on changing people, is much more demanding. We can cultivate rationality with trainings focused on a particular single competence, such as statistical reasoning (Sirota, Kostovičová, \& Vallée- 
Tourangeau, 2015) and considering alternatives (Hirt\&Markman, 1995), or we can use a complex intervention. Morewedge et al. (2015), for instance, reduced crucial cognitive biases via teaching a whole set of competences, such as hypothesis testing, considering countervailing evidence, alternative explanations and perspectives, base rates and anchors, and encouraging people to reconsider their initial answers. There are, however, some indirect methods as well, mindfulness meditation being one of them (Hafenbrack, Kinias, \& Barsade, 2014). Another indirect debiasing intervention is based on mental simulation.

During the process of mental simulation people think of various relevant but potentially converse alternatives (Galinsky \& Moskowitz, 2000). In the naturalistic decision-making paradigm, mental simulation is a conscious, deliberate, and analytic strategy used to evaluate different courses of action (Klein, 2008). It can be trained or primed. In a single-shot intervention, mental simulation can be induced with a counterfactual priming (Galinsky \& Moskowitz, 2000). The method is based on a scenario, which encourages people to produce counterfactuals - i.e. thoughts of what might have been, e.g., "If only I had tried more ...".

They represent alternative realities for a past event (e.g., "... I would have been better off."). Taken together, counterfactual scenarios are supposed to activate a mental simulation mindset in which various alternatives are considered (Galinsky \& Moskowitz, 2000). As a result, mental simulation might affect cognitive performance in situations when taking into account different views and explanations is crucial. Indeed, it has been found to mitigate confirmation bias in both individuals (Galinsky \& Moskowitz, 2000) and groups (Kray \& Galinsky, 2003), to enhance performance in the hidden profile task (Liljenquist, Galinsky, \& Kray, 2004), and to reduce prejudice (Miller, Markman, Wagner, \& Hunt, 2013).
Here we decided to test the effect of counterfactual priming on the three components of biased reasoning: selectively seeking hypothesisconfirming evidence, neglecting alternative explanations and unwillingness to reconsider the default option in response to new information. Biased search for information in line with prior hypothesis or belief is a key component of the confirmation bias, one of the most robust cognitive deviations (Nickerson, 1998). Neglecting alternative explanations is closely tied to many cognitive biases. One of many examples is the fundamental attribution error, manifested as overestimation of internal factors and underestimation of external factors when evaluating other people's behavior (Ross, 1977). The last one, sticking with the default, is at the heart of status-quo bias - the tendency to prefer the current state of affairs despite the existence of better alternatives (Samuelson \& Zeckhauser, 1988). These three tendencies are in sharp contrast to open-minded thinking (Baron, 1993) and, therefore, represent barriers to rational judgment and decision-making (e.g., Svedholm-Häkkinen \& Lindeman, 2018).

Besides examining the effect of mental simulation on biased reasoning, we were also interested in its explanation, which has yet to be empirically tested. In the context of the dualprocesses account of human reasoning (e.g., Wason \& Evans, 1975), mental simulation is believed to correspond to the System 2 processes (Klein, 2008). In line with this assumption, counterfactual thinking enhanced performance on analytic problems (Kray, Galinsky, \& Wong, 2006; Markman, Lindberg, Kray, \& Galinsky, 2007). We, therefore, hypothesized that the effect of mental simulation on tasks which require both overriding automatic responses and considering alternatives is linked to activation and inhibition of the two Systems. Specifically, we expected that mental simulation induced with a counterfactual scenario would support the analytic System 2 processes and 
inhibit the intuitive System 1 processes, and this would result in less biased reasoning. As a proxy for intuitive versus analytic thinking we chose cognitive reflection. A high score on the cognitive reflection tests (Frederick, 2005; Sirota, Kostovičová, Juanchich, Dewberry, \& Marshall, 2018; Toplak, West, \& Stanovich, 2014) means that the person is able to exert cognitive effort, suppress their automatic incorrect responses, and engage in deliberative thinking. It is a very strong predictor of susceptibility to cognitive biases (e.g., Toplak, West, \& Stanovich, 2011, 2014), rational choices and reallife decision outcomes (e.g., Campitelli \& Labollita, 2010; Juanchich, Dewberry, Sirota, \& Narendran, 2016).

Taken together, we aimed at examining whether counterfactual priming would mitigate biased reasoning via changes in cognitive reflection. We anticipated that thanks to mental simulation, activated with a counterfactual scenario, people would inhibit their intuition, engage in analytic thinking, and their responses would be less biased as a result. Specifically, they would seek both hypothesis-confirming and disconfirming evidence, they would consider alternative explanations, and would be willing to reconsider the default option when exposed to novel information.

\section{Method}

\section{Participants}

We assumed a counterfactual priming effect similar to the one in Galinsky and Moskowitz's (2000) study, $d=0.55$. Based on a power calculation (two-sided tests, $\alpha=.05,1-\beta=.95$ ), we planned to recruit at least 314 participants in total (adjusted for the assumption that one fifth would fail to pass an attention check item). Four hundred eighty-six people in a convenience sample filled out our online questionnaire. After excluding those who failed to pass the at- tention check item, our final sample consisted of 462 participants $(60 \%$ female, 18 to 59 years, $M=24.3, S D=6.8$ ). Two thirds were university students. Concerning the study programs, the participants were students or graduates in natural and technical sciences (48\%), social sciences and humanities (40\%) or other fields (art, sport, etc.; $12 \%)$.

\section{Design and Procedure}

The participants first answered basic sociodemographic items. Afterwards, they solved a first series of cognitive reflection items (CRT1). Then, they were randomly redirected to one of six groups, as depicted in Figure 1. These groups represent three control groups (CG): CG for Task 1, CG for Task 2, and CG for Task 3. After solving one of the three tasks, all the participants proceeded to our experimental manipulation - the counterfactual priming. Next, we asked them to solve one of the remaining two tasks. For instance, roughly half of those who solved Task 3 prior to the intervention, were administered Task 1 after the priming. The other half solved Task 2 . Taken together, there were six groups: Task 1 - Priming - Task 2, Task 1 Priming - Task 3, Task 2 - Priming - Task 1, etc. As a result, there was a different composition of the control and the experimental group for each of the three tasks. Finally, the participants answered a second series of cognitive reflection problems (CRT2). The two CRT tests were administered in a randomized order. Priming exposure acted as an independent variable, performance in the three tasks as dependent variables, and correct and intuitive CRT scores (control group: CRT1 / experimental group: CRT2) as mediators.

\section{Materials and Measures}

We conducted two pilot studies in the first phase of the research to empirically evaluate 


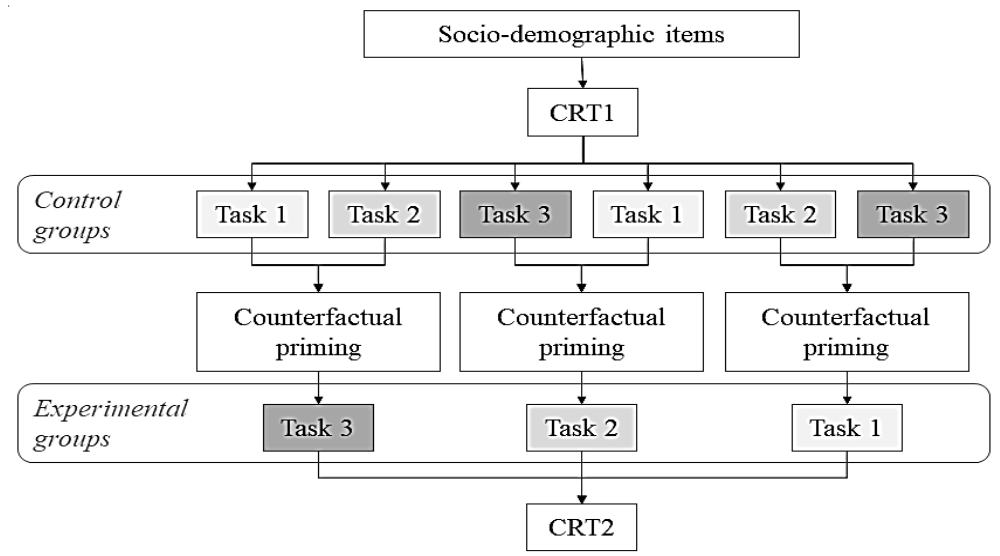

Task 1: Selectively seeking hypothesis-confirming evidence

Task 2: Neglecting alternative explanations

Task 3: Unwillingness to reconsider the default option

Figure 1 The design and procedure used in the experiment

stimuli and material. First, we adopted a counterfactual scenario from Galinsky and Moskowitz (2000) and pretested it with a sample of 39 participants. The story concerned Jane, a woman at a concert of her favorite band. She switched her seat to have a better view but, as a result, she did not win a trip to Hawaii since the winner was sitting exactly at the place she had left. The other, neutral, scenario did not contain this outcome - the winning seat had nothing to do with Jane's choice. The participants were asked to generate thoughts that might run through Jane's head after the concert. Answers of the two groups (neutral vs. counterfactual scenario) were coded by two independent raters. Given a high reliability, $r=$ $.82, p<.001$, the pairs of ratings were averaged. The neutral scenario produced significantly less counterfactual thoughts than the counterfactual scenario $(M=0.3, S D=0.4$ vs. $M=0.8$, $S D=0.7$, respectively), $t(37)=-2.73, p=.010$, $d=0.88$. With respect to the results and in order to increase the effectiveness of the manipula- tion, we added a sentence at the end of the scenario: "On her way home, Jane is thinking of what she would have gained and what she would have lost if she had not switched her seat".

The objective of the other pilot study $(N=$ 26) was to prepare two short cognitive reflection tests (CRT) of roughly the same difficulty. We tested the original CRT tasks (Frederick, 2005), their alternatives with a modified wording (Kostovičová, Dudeková, \& Konečný, 2013), new items from the extended version (Toplak et al., 2014), and verbal CRT problems (Sirota et al., 2018). We checked the items for their difficulty, discrimination power, content clarity and familiarity. Based on the results, we designed two versions, consisting of 2 verbal and 2 numerical tasks, for instance: "How many of each animal did Moses take on the ark?" (Sirota et al., 2018) or "Jerry received both the 15 th highest and the 15 th lowest mark in the class. How many students are in the class?" (Toplak et al., 2014, p. 151). Performance on the two tests was almost identical (CRTa: $M=1.6$, 
$S D=1.0$; CRTb: $M=1.6, S D=0.9), t(25)<0.01$, $p=1.000, d<0.01$. We coded the answers as correct, intuitive or other. The scores of correct as well as intuitive responses ranged from 0 to 4.

The indicators of biased reasoning focused on selectively seeking hypothesis-confirming evidence (Task 1), neglecting alternative explanations (Task 2), and unwillingness to reconsider the default option in response to new information (Task 3). The first task was inspired by Galinsky and Moskowitz (2000). The story was about Tereza who attends speed-dating and before the event starts, she spots a man whose behavior suggests he is an introvert. Since she knows she will have only one minute to talk to him, she wants to prepare some questions to test whether her impression is correct. The participants were asked to choose 5 out of 10 questions. Half of the questions captured typical behavior of an introvert. These five "introverted" questions were supposed to elicit hypothesis-confirming answers (e.g., "Do you like spending evenings at home?"). The content of the other five questions concerned typical behavior of extroverts. These five "extroverted" questions were meant to elicit hypothesis-disconfirming answers (e.g., "Do you like meeting new people?"). The more introverted questions and the fewer extroverted questions a participant chooses, the more s/he seeks information that would support the initial hypothesis of the man being an introvert. Thus, the difference between the number of introverted and extroverted questions (range: -5 to $5 ;-5$ : extroverted questions only / +5 : introverted questions only) captures the level, to which a person selectively seeks hypothesis-confirming evidence.

In the second task, the participants were asked to imagine that they have a new colleague, Adam. He seems hardworking, diligent, and punctual, yet his mood and behavior are strange and unpredictable. After the scenario, which provided both reasons for situation- and personality-based explanations of Adam's actions, the participants answered three questions on 7-point Likert scales. The first two items were only distractors and concerned their overall impression and their view on the colleague's potential. The third, crucial one focused on the explanation of the colleague's unstable behavior (-3: situational factors only; 3: colleague's personality only). We were interested in people's preference for one or the other category (situation vs. personality), but our main focus was on the absolute score (range 0 to 3 ). Thus, given the doubts on the robustness of the fundamental attribution error (e.g., Malle, 2006), and since human behavior is generally influenced by both internal and external factors, and the scenario did not encourage preference for any of them, the participants should have considered both explanations. Thus, the higher the score the more a person neglects an alternative explanation.

The last task was about vaccination against HPV (human-papillomavirus, the main cause of cervical cancer). The participants were informed that this vaccine is not mandatory in Slovakia. After being provided with arguments on possible benefits of the vaccination, and on the risks of side-effects as well (Kostovičová, Bašnáková, \& Bačová, 2017), they answered two questions. The first one concerned whether vaccination is one of the greatest discoveries of medicine or rather one of its most controversial topics. In the other - critical one, the participants expressed whether they would be willing to reconsider the default option (non-mandatory vaccination) in response to new information provided (no/yes).

\section{Results}

First of all, scores of the correct CRT answers in the two measurements were moderately correlated, $r=.41, p<.001$, and so were the scores 
of the intuitive answers, $r=.36, p<.001$. The numbers of correct CRT answers, $r=-.19, p=$ .001 , and intuitive CRT responses, $r=.16, p=$ .006 , in the second measurement correlated with selectively seeking hypothesis-confirming evidence. We found no significant correlation between the three indicators of biased reasoning.

There was no substantial effect of experimental manipulation on correct CRT responses, regardless of the specific order. Overall, the CRT scores before and after intervention were very similar $(M=2.0, S D=1.2$ vs. $M=1.9, S D=1.2)$, $t(461)=1.54, p=.324, d=0.10$. However, counterfactual priming reduced intuitive $C R T$ responses $(M=1.3, S D=1.2$ vs. $M=1.0, S D=$ $1.0), t(461)=6.04, p<.001, d=0.40$. It also enhanced the number of other, incorrect but non-intuitive, answers $(M=0.7, S D=0.9$ vs. $M=1.2, S D=1.2), t(461)=-7.24, p<.001, d=$ 0.48 . These patterns were the same when we separately analyzed changes in performance in verbal and numerical CRT problems. Taken together, mental simulation prompted with a counterfactual scenario helped override automatic intuitive responses but failed to sufficiently help the participants to come up with correct solutions.

At the same time, responses in the third task remained unaffected. Specifically, two thirds of the control $(66 \%, n=107)$ and the experimental group $(68 \%, n=101)$ expressed that vaccina- tion is one of the greatest discoveries of medicine rather than one of its most controversial topics, $\chi^{2}(1)=0.11, p=.745, \varphi=.02$. And, more importantly, willingness to reconsider the default option was also almost identical in the control $(44 \%, n=90)$ and the experimental group $(43 \%, n=85), \chi^{2}(1)=0.07, p=.791, \varphi=.02$. Correlation between the two responses was significant, yet weak, $r_{S}=.22, p<.001$.

As for the assessment of the colleague's behavior, responses of the experimental group were more in line with the situational explanation $(M$ Rank $=146.0)$ compared to the control group $(M \operatorname{Rank}=159.3)$, yet the difference was nonsignificant, $M-W U=10538.5, p=.175, r_{m}=0.08$. When comparing the level to which one neglects an alternative explanation, we found that the score was significantly higher in the control group $(M=1.8, S D=1.0$ vs. $M=1.4, S D=$ $1.0), t(302)=3.55, p<.001, d=0.41$. Thus, counterfactual priming enhanced considering alternative explanations.

Comparisons of the numbers of introverted and extroverted questions (range 0 to 5), and the overall score (range -5 to 5) for the "speeddating" task are presented in Figure 2. The number of hypothesis-confirming (introverted) questions was slightly higher in the control group, but without a significant difference, $t(307)=1.76, p=.080, d=0.20$. The number of hypothesis-disconfirming (extroverted) ques-

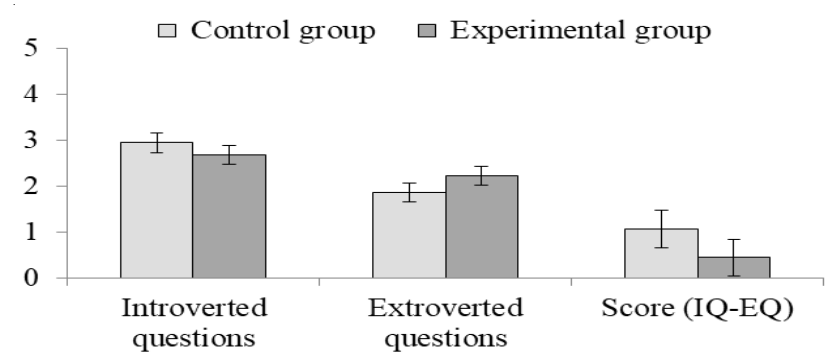

Figure 2 Comparison of hypothesis dis/confirming choices 
tions was significantly higher in the experimental group, $t(307)=-2.43, p=.016, d=0.28$. And finally, the overall score was significantly higher in the control group, $t(307)=2.17, p=.031, d=$ 0.25 , which means that counterfactual priming reduced selectively seeking hypothesis-confirming evidence.

Since the counterfactual priming did not affect correct CRT responses and willingness to reconsider the default option, we performed two mediation analyses (Hayes, 2013) with priming as an independent variable and intuitive CRT responses as a mediator. In the first tested model with considering alternative explanations as a dependent variable, only the effects of intervention were significant. The other tested model with seeking hypothesis-confirming evidence is depicted in Figure 3. We found that the number of intuitive CRT answers mediated the effect of counterfactual priming on selectively seeking hypothesis-confirming evidence. Yet, the indirect effect $(-0.083)$ and the $R$-squared mediation effect size (.004) were quite small. Order of the two CRT versions was entered as a covariate.

\section{Discussion}

Cognitive errors are costlyand are getting costlier; therefore, the main challenge for the current judgment and decision-making research is devel- opment of improvement strategies (Milkman, Chugh, \& Bazerman, 2009). Debiasing efforts can be focused either on modification of the environment (such as helpful external representations) or the decision makers themselves (such as cultivating rationalityvia training). Both approaches have their pros and cons but - except for some nudge techniques - their implementation in the real-world settings poses many pragmatic difficulties and requires a lot of time and effort. Previous research showed that mental simulation prompted with a counterfactual scenario represents a "fast and frugal" way of reducing biases for which considering alternatives is crucial (Galinsky\& Moskowitz, 2000; Kray\& Galinsky, 2003; Liljenquist etal., 2004; Miller etal., 2013). Given the link between higher performance in analytic problems and counterfactual thinking (Kray et al., 2006; Markman et al., 2007), we hypothesized that the effect of mental simulation on biased reasoning would be mediated by changes in cognitive reflection. In other words, we anticipated that exposure to counterfactual scenario would result in lower levels of seeking hypothesis-confirming evidence, ignoring alternative explanations, and unwillingness toreconsider the default option thanks to inhibiting intuitive System 1 processes and activating deliberative System 2 thinking.

We found that counterfactual priming failed to enhance the cognitive reflection scores, yet

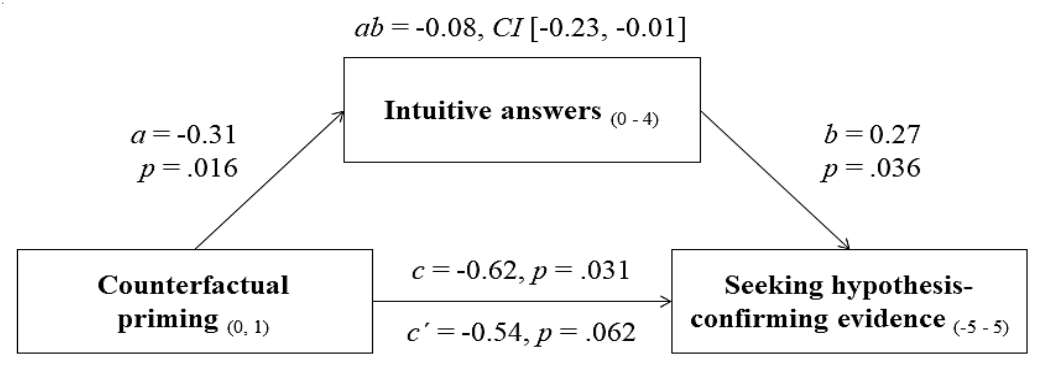

Figure 3 Intuition inhibition as a mediator of the effect of counterfactual priming on selectively seeking hypothesis-confirming evidence 
it reduced the level of intuitive responses. This means that mental simulation mindset helped in suppressing appealing automatic answers but was not efficient in generating the correct ones instead. As Stanovich (2018) points out, some cognitive errors cannot be unambiguously credited to miserly processing. Thus, sometimes people miss some important mindware component, such as knowledge of scientific and probabilistic reasoning, or math skills. Given the strong association of the CRT with numeracy (e.g., Liberali et al., 2012), cognitive reflection performance might represent a quite stable individual disposition, which is rather difficult to change (e.g., Stagnaro, Pennycook, \& Rand, 2018). Yet, a positive effect of mental simulation on intuition inhibition is a promising finding, which deserves further examination.

The other case, which contradicted our assumptions, is finding no effect of the experimental manipulation on the willingness to reconsider the default option in response to new information. We suppose the explanation is twofold. First, we chose a quite controversial and emotionally charged topic, which polarizes society into two opposite groups, one of which is significantly smaller in number (Masaryk \& Hatoková, 2016; Tvardzík, 2015). Thus, people's preferences might be so strong that they are resistant to such a simple debiasing method. This is in line with our previous findings on domain specificity of the effects of counterfactual thinking (Strachanová \& Grežo, 2018). In addition, maybe the participants refused to reconsider the default option not because they are against vaccination as such but because we used the term "mandatory". Next time we would replace the forced binary choice with a Likert scale as well. We also plan to further investigate the moderating role of the domain in debiasing via mental simulation.

On the other hand, counterfactual priming substantially facilitated search for hypothesisdisconfirming evidence and consideration of alternative explanations. These patterns suggest that mental simulation might help in overcoming cognitive failures such as belief bias, confirmation bias, fundamental attribution error, myside bias, self-serving bias, survivorship bias, and many more. It is even more promising when we take into account that counterfactual thinking can be systematically trained (Hendrickson, 2008), so it becomes a natural component of one's cognitive strategy repertoire. Mental simulation toward future, for instance, is a typical supporting strategy in naturalistic decision-making (Klein, 2008; Klein et al., 2003). Counterfactual thinking might therefore become a part of training for managers, teachers and other professionals, and can be utilized in educational campaigns. In order to determine whether it is appropriate to use counterfactuals in direct or indirect debiasing methods, we need to explore the nature of the bias (Kahn, Luce, \& Nowlis, 2006). Most importantly, we have to know whether the decision maker is aware of the underlying processes leading to the bias. Since we believe that the three components of biased reasoning we have focused on in our research are largely subconscious, indirect debiasing method is a proper strategy for their reduction. Although the effects of counterfactual priming are short-term only, indirect debiasing strategies eliminate some of the barriers associated with direct interventions, such as bias blind spot or perceived relevance (Lilienfeld, Ammirati, \& Landfield, 2009).

The current study has several limitations, out of which two were already mentioned: CRT as a problematic measure of analytic (or intuitive) reasoning, and the task with mandatory vaccination and a binary question as a problematic measure of willingness to reconsider the default option. Instead of a Likert scale, an open question for participants to state their own reasons for a colleague's behavior might produce more ecologically valid answers in the task on 
neglecting alternative explanation. In the last task on selectively seeking hypothesis-confirming evidence, a few neutral questions (neither introverted nor extroverted) might help as distractors. Overall, multiple tasks to measure the same aspect of biased reasoning and composite scores would ensure results of a higher reliability. We also admit that our sample was not representative, especially in terms of age and education. And, most importantly, we cannot consider our search for explanation of debiasing power of mental simulation successful. Although intuition inhibition mediated the effect of counterfactual priming on seeking hypothesis-confirming evidence, the indirect effect was rather small and the finding is inconclusive.

Thus, future research should address the missing parts of the "counterfactual priming" puzzle. First, the mediators of the effect: either analytic thinking measured in a different way, or some other constructs that are linked to biased reasoning and cognitive performance, such as open-mindedness (e.g., Svedholm-Häkkinen \& Lindeman, 2018) or metacognitive awareness (Schraw, 1998). Future research should focus on moderating variables as well. These might be some personal characteristics which make people less or more resistant to the intervention, and task features as well. According to the recent neuroscience findings (De Brigard, Spreng, Mitchell, \& Schacter, 2015), for instance, effectiveness of the counterfactual priming might depend on whether the protagonist in the scenario is an unknown person or the participant. Another moderator worthy of investigation is mental simulation toward past versus future. Follow-up studies could also focus on particular cognitive biases, which have not been tested under the mental simulation condition. Last but not least, the effect of mental simulation as both indirect and direct debiasing strategy should be tested in the realworld settings.

\section{References}

Baron, J. (1993). Why teach thinking? An essay. Applied Psychology: An International Review, 42(3), 191-214. doi: 10.1111/j.1464-0597.1993. tb00731.x

Benson, B. (2016). Cognitive bias cheat sheet. Retrieved from https://betterhumans.coach.me/cognitive-bias-cheat-sheet-55a472476b18

Campitelli, G., \& Labollita, M. (2010). Correlations of cognitive reflection with judgments and choices Judgment and Decision Making, 5(3), 182-191.

De Brigard, F., Spreng, N. R., Mitchell, J., \& Schacter, D. (2015). Neural activity associated with self, other, and object-based counterfactual thinking. NeuroImage, 109, 12-26. doi: 10.1016/j. neuroimage.2014.12.075

Fischhoff, B. (1982). Debiasing. In D. Kahneman, P. Slovic, \& A. Tversky (Eds.), Judgment under uncertainty: Heuristics and biases (pp. 422-444). Cambridge: Cambridge University Press.

Frederick, S. (2005). Cognitive reflection and decision making. Journal of Economic Perspectives, 19(4), 25-42. doi: 10.1257/089533005775196732

Hafenbrack, A. C., Kinias, Z., \& Barsade, S. G. (2014). Debiasing the mind through meditation: Mindfulness and the sunk-cost bias. Psychological Science, 25(2) 369-376. doi: 10.1177/0956797613503853

Hayes, A. F. (2013). An introduction to mediation, moderation, and conditional process analysis: A regression-based approach. New York, NY: Guilford Press.

Hirt, E., \& Markman, K. (1995). Multiple explanation: A consider-an-alternative strategy for debiasing judgments. Journal of Personality and Social Psychology, 69(6), 1069-1086. doi: 10.1037/00223514.69.6.1069

Galinsky, A. D., \& Moskowitz, G. B. (2000). Counterfactuals as behavioral primes: Priming the simulation heuristic and consideration of alternatives. Journal of Experimental Social Psychology, 36(4), 384-409. doi: 10.1006/jesp.1999.1409

Hendrickson, N. (2008). Counterfactual reasoning. A basic guide for analysts, strategists, and decision makers. Washington, D.C.: The National Intelligence University.

Juanchich, M., Dewberry, C., Sirota, M., \& Narendran, S. (2015). Cognitive reflection predicts reallife decision outcomes, but not over and above personality and decision making styles. Journal of Behavioral Decision Making, 29(1), 52-59. doi: 10.1002/ bdm. 1875

Kahn, B. E., Luce, M. F., \& Nowlis, S. M. (2006). Debiasing insights from process tests. Journal of 
Consumer Research, 33(1), 131-138. doi:10.1086/ 500492

Klein, G. (2008). Naturalistic decision making. Human Factors, 50(3), 456-460. doi: 10.1518/ $001872008 \times 288385$

Klein, G., Ross, K. G., Moon, B. M., Klein, D. E., Hoffman, R. R., \& Hollnagel, E. (2003) Macrocognition. IEEE Intelligent Systems, 18(3), 81-85. doi: 10.1109/MIS.2003.1200735

Kostovičová, L., Bašnáková, J., \& Bačová, V. (2017). Predicting perception of risks and benefits within novel domains. Studia Psychologica, 59(3), 176192 .

Kostovičová, L., Konečný, M., \& Dudeková, K. (2013). Čo to vlastne meriame? Reflexia Testu kognitívnej reflexie [What do we actually measure? Reflection of the Cognitive Reflection Test]. In I. Sarmány-Schuller (Ed.), Otázniky rozhodovania: teória, empíria, život, 31. Psychologické dni: zbornik príspevkov [31st Psychological days: Conference proceedings] (pp. 166-171). Bratislava: STIMUL.

Kray, L. J., \& Galinsky, A. D. (2003). The debiasing effect of counterfactual mind-sets: Increasing the search for disconfirmatory information in group decisions. Organizational Behavior and Human Decision Processes, 91(1), 69-81. doi: 10.1016 S0749-5978(02)00534-4

Kray, L. J., Galinsky, A. D., \& Wong, E. (2006). Thinking within the box: The relational processing style elicited by counterfactual mind-sets. Journal of Personality and Social Psychology, 91(1), 33-48. doi: 10.1037/0022-3514.91.1.33

Lerner, J. S., \& Tetlock, P. E. (1999). Accounting for the effects of accountability. Psychological Bulletin, 125(2), 255-275. doi:10.1037/0033-2909.125. 2.255

Liberali, J. M., Reyna, V. F., Furlan, S., Stein, L. M., \& Pardo, S. T. (2012). Individual differences in numeracy and cognitive reflection, with implications for biases and fallacies in probability judgment. Journal of Behavioral Decision Making, 25(4), 361381. doi: $10.1002 / \mathrm{bdm} .752$

Lilienfeld, S. O., Ammirati, R., \& Landfield, K. (2009). Giving debiasing away: Can psychological research on correcting cognitive errors promote human welfare? Perspectives on Psychological Science, 4(4), 390-398. doi: 10.1111/j.1745-6924.2009.01144.x

Liljenquist, K. A., Galinsky, A. D., \& Kray, L. J. (2004). Exploring the rabbit hole of possibilities by myself or with my group: The benefits and liabilities of activating counterfactual mindsets for information sharing and group coordination. Journal of Behavioral Decision Making, 17(4), 263-279. doi: $10.1002 / \mathrm{bdm} .479$
Malle, B. F. (2006). The actor-observer asymmetry in attribution: A (surprising) meta-analysis. Psychological Bulletin, 132(6), 895-919. doi:10.1037/00332909.132.6.895

Markman, K. D., Lindberg, M. J., Kray, L. J., \& Galinsky, A. D. (2007). Implications of counterfactual structure for creative generation and analytical problem solving. Personality and Social Psychology Bulletin, 33(3), 312-324. doi: 10.1177/ 0146167206296106

Masaryk, R., \& Hatoková, M. (2016). Qualitative inquiry into reasons why vaccination messages fail. Journal of Health Psychology, 22(14), 1880-1888. doi: $10.1177 / 1359105316656770$

Milkman, K. L., Chugh, D., \& Bazerman, M. H. (2009). How can decision making be improved? Perspectives on Psychological Science, 4(4), 379-383. doi: 10.1111/j.1745-6924.2009.01142.x

Miller, A. K., Markman, K. D., Wagner, M. M., \& Hunt, A. N. (2013). Mental simulation and sexual prejudice reduction: The debiasing role of counterfactual thinking. Journal of Applied Social Psychology, 43(1), 190-194. doi: 10.1111/j.15591816.2012.00992.x

Morewedge, C. K., Yoon, H., Scopelliti, I., Symborski, C. W., Korris, J. H., \& Kassam, K. S. (2015). Debiasing decisions: Improved decision making with a single training intervention. Policy Insights from Behavioral and Brain Sciences, 2(1), 129-140. doi: $0.1177 / 2372732215600886$

Nickerson, R. S. (1998). Confirmation bias: A ubiquitous phenomenon in many guises. Review of General Psychology, 2(2), 175-220. doi: 10.1037/10892680.2.2.175

Ross, L. (1977). The intuitive psychologist and his shortcomings: Distortions in the attribution process. Advances in Experimental Social Psychology, 10, 173-220. doi: 10.1016/S0065-2601(08)603573

Samuelson, W., \& Zeckhauser, R. (1988). Status quo bias in decision making. Journal of Risk and Uncertainty, 1(1), 7-59. doi: 10.1007/BF00055564

Schraw, G. (1998). Promoting general metacognitive awareness. Instructional Science, 26(1), 113-125. doi: 10.1023/A:1003044231033

Sirota, M., Kostovičová, L., \& Juanchich, M. (2014). The effect of iconicity of visual displays on statistical reasoning: evidence in favor of the null hypothesis. Psychonomic Bulletin \& Review, 21(4), 961968. doi: 10.3758/s13423-013-0555-4

Sirota, M., Kostovičová, L., Juanchich, M., Dewberry, C., \& Marshall, A. C. (2018). Measuring cognitive reflection without math: Developing and validating verbal cognitive reflection test. Manuscript submitted for publication. 
Sirota, M., Kostovičová, L., \& Vallée-Tourangeau, F. (2015). How to train your Bayesian: A problem-representation transfer rather than a format-representation shift explains training effects. The Quarterly Journal of Experimental Psychology, 68(1), 1-9. doi: 10.1080/ 17470218.2014 .972420

Soll, J. B., Milkman, K. L., \& Payne, J. W. (2016). A user's guide to debiasing. In G. Keren \& G. Wu (Eds.), The Wiley Blackwell handbook of judgment and decision making II (pp. 924-951). Malden, MA: Wiley-Blackwell.

Stanovich, K. E. (2018). Miserliness in human cognition: The interaction of detection, override and mindware. Thinking \& Reasoning, 24(4), 423-444. doi: 10.1080/13546783.2018.1459314

Stagnaro, M. T., Pennycook, G., \& Rand, D. G. (2018). Performance on the Cognitive Reflection Test is stable across time. Judgment and Decision Making, 13(3). doi: /10.2139/ssrn.3115809

Strachanová, D., \& Grežo, M. (2018). Keby som sa rozhodol inak: Kontrafaktové myslenie a efekt utopených nákladov $\mathrm{v}$ rozhodovaní [If I had decided differently: Counterfactual thinking and the sunk-cost effect in decision-making]. In E. Maierová, L. Viktorová, J. Suchá, \& M. Dolejš (Eds.), PhD existence 2018 - Nekonečno v psychologii: Sbornik př́spěvků [PhD existence 2018 - Infinity in psychology: Conference pro- ceedings] (pp. 97-103). Olomouc: Univerzita Palackého.

Svedholm-Häkkinen, A. M., \& Lindeman, M. (2018). Actively open-minded thinking: Development of a shortened scale and disentangling attitudes towards knowledge and people. Thinking \& Reasoning, 24(1), 21-40. doi: 10.1080/13546783.2017.1378723

Thaler, R. H., \& Sunstein, C (2008). Nudge. Yale University Press.

Toplak, M. E., West, R. F., \& Stanovich, K. E. (2011). The Cognitive Reflection Test as a predictor of performance on heuristics and biases tasks. Memory \& Cognition, 39(7), 1275-1289. doi: 10.3758/s13421011-0104-1

Toplak, M. E., West, R. F., \& Stanovich, K. E. (2014). Assessing miserly information processing: An expansion of the Cognitive Reflection Test. Thinking \& Reasoning, 20(2), 147-168. doi: 10.1080/ 13546783.2013 .844729

Tvardzík, J. (2015, May 20). Očkovanie: Kolektívna imunita je $\mathrm{v}$ ohrození. Epidémia je reálna [Vaccination: Collective immunity is at risk. The epidemic is real]. Trend. Retrieved from https://www.etrend.sk/ ekonomika/ockovanie-kolektivna-imunita-je-vohrozeni-epidemia-je-realna-varujeexpertka.html? split=1

Wason, P. C., \& Evans, J. S. B. T. (1975). Dual processes in reasoning? Cognition, 3(2), 141-154. doi: 10.1016/0010-0277(74)90017-1 